\title{
Construction of Cloud Library
}

\author{
Dongju $\mathrm{Bu}^{1}$ and Yueheng $\mathrm{Cao}^{2}$ \\ ${ }^{1}$ Library of Changchun University of Science and Technology, Changchun, Jilin Province, China \\ ${ }^{2}$ School of management, Jilin University, Changchun, Jilin Province, China
}

\begin{abstract}
Cloud library is a new type of Digital Library based on the network platform. It is a collection of all kinds of digital library. Through the middleware technology and virtualization technology, the establishment of a strong academic digital resources literature service model, unified distribution, management, monitoring, deployment and backup resources. User application service platform resources, database resources, storage resources and service system, etc., to support the operation of information resources construction platform.
\end{abstract}

Keywords-cloud computing; cloud service platform; sharing model; architecture model design

\section{INTRODUCTION}

Cloud library cloud computing technology is applied to the management of library services, cloud computing technology to integrate the library resources to be integrated in the computer. Users through the search engine to obtain the necessary resources, can be used to pay. Cloud computing services platform is applied to the library's resource management, cloud computing providers to integrate the collection of books, journals and other documents and information, to share the information resources of the library . Users can access the Internet to obtain the necessary information resources, enjoy convenient services.

\section{OVERVIEW OF CLOUD LIBRARY}

\section{A. Cloud Computing Technology}

Cloud computing is a new network resource model based on parallel processing, distributed processing, grid computing, and the Internet. Cloud computing is a computing program through the network to split into numerous smaller subroutines, calculation processing program distributed in a large number of computers, make full use of Internet resources, the establishment of a more powerful function computing center. Cloud computing is a new business computing model that can be shared on the basis of architecture. Cloud computing is the development of parallel computing, distributed computing and grid computing. Cloud computing virtualization, utility computing infrastructure as a service (Iaa S), Paa S (platform as a service), Saa $\mathrm{S}$ (software as a service) the concept of mixed results, as shown in Figure 1.

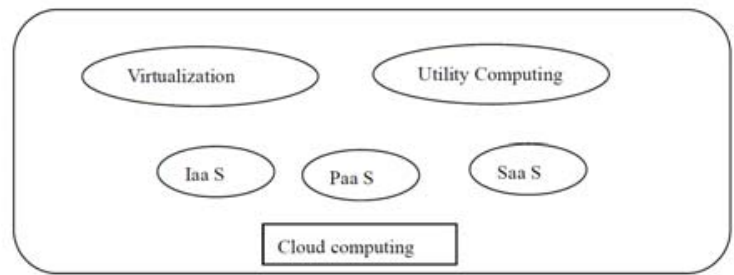

FIGURE I. CLOUD COMPUTING CONCEPT MODEL

\section{B. Cloud Library}

The Cloud library refers to the library to borrow books, query through the network, and the use of the Internet will be many in the library of data and literature, combining with the resources, the development of library service network, with the mobile phone terminal, providing fast intelligent service for the user. In order to realize the combination of the library system and the information system, and improve the service function of the library. Cloud library is a new type of Digital Library Based on the network platform. It is a collection of all kinds of digital library. Through the middleware technology and virtualization technology, build digital resources services model strong discipline, unified allocation and management, monitoring, deployment and backup resources, user application service platform resources, database resources, storage resources and service system and so on, to support the construction of information resources platform operation.

Cloud computing ability of super large library, storage space, storage will automatically update the data at any time, a task into multiple sub tasks, combined with data processing, to extract user needed information, improve the efficiency of library services, to meet the needs of users. Cloud library intelligent management system to provide users with multi node task transfer services, real non broken security services, so that data security and reliability, improve service quality. Building cloud library information system (CLMIS) platform, the integration of data and resources, the establishment of a shared environment, providing a convenient deep open mobile information services. According to the characteristics of cloud computing, as well as the needs of users, the CLMIS can be divided into user layer, application layer, management layer, data layer, physical layer. The physical layer is the basis of the whole architecture, providing the host, memory, network and other virtual hardware resources. Data layer for collecting, updating, scheduling, backup and other scattered nodes cloud data resources. Add and delete users, management of process scheduling, quality of service, distributed computing cluster, parallel program design, data storage, concurrency control etc.. Application layer provides user login, verification, 
cancellation, resource promotion and other related services. The user layer is the main body of CLMIS, including login, authentication, access to the system, the implementation of related operations, the relevant service.

\section{Characteristics of Cloud Library}

Cloud Library in the Internet, web, Web2.0, entered the era of cloud computing, the user's needs are open, professional, dynamic, integrated features.

- Provide readers with an information access platform

Cloud library has shared, to meet the needs of a variety of projects. Users to borrow and retrieval more convenient and flexible, through the network terminal to enter the library to check, browse, download and print information. System login entrance through the library to provide the system extension application interface. To achieve a one-stop search, the reader of a one-time search condition, you can query the entire Internet resources, access to the required resources through links.

- Makes the library resource allocation more efficient

Cloud library to improve the description of resource metadata information, editing and browsing. More than one server to form a computer group cooperative work, has a very powerful storage capacity and computing power, complete a variety of complex work, information storage is very strong. Cloud library services do not have the time and space constraints, the user can obtain the required resources in any terminal. Cloud library is able to integrate the information resources and services of the library as a whole, and integrate the information resources of different sources and different protocols, and seamlessly connect the information resources. Through the integration of systems, technologies, protocols, databases and retrieval methods, the formation of cross platform, cross database and cross content of the information resource database, with integrated retrieval function, the query speed is faster, the use of very flexible.

\section{- $\quad$ Reduce the operating cost of the library}

Cloud library services according to the needs of users to charge a fee for the integration of distributed resources, improve the search capability, reduce the cost of library construction, to achieve a low cost, high efficiency of quality services. Cloud library has a strict authority management, users can carry out data sharing, users pay a small amount of cost, to achieve better results.

\section{- Improve the information security of the library}

Cloud library to add library database network firewall, the development of passwords or face recognition and authentication technology, the development of confidentiality laws and regulations. For electronic books, data transmission, the article reproduced, browse and so on all the possible causes of intellectual property issues into account. Cloud Library Based on the Internet data storage capabilities, data can be transmitted simultaneously, the user's data resources are more secure and reliable.

\section{CONSTRUCTION OF CLOUD LIBRARY}

\section{A. Cloud Library Infrastructure}

The base layer of the architecture is the library cloud infrastructure as a service, provide virtual hardware resources, including database management, network, storage, virtual host, decision of cloud library service capacity and service scope. The infrastructure layer will be a lot of resources on the network nodes, workstation, PC, database, large storage devices, the virtualization technology for access to the network, realize the connection of multiple node resources. In order to ensure the information service needs, realize the optimization of cloud services cloud platform library settings, virtual cloud computing services platform to ensure the quality of service. Cloud library node platform can increase or cut work, which is conducive to the overall operation of the platform.

\section{B. Cloud Library Data Tier}

Data security, data encryption and backup, data processing, data processing, data distribution, data scheduling and management. The use of virtualization technology and middleware technology, resource allocation and scheduling of cloud library, user service request, operated by the service interface, through reasonable analysis, announced to the node platform cloud library. Cloud library node platform information, after obtaining the integration of operations, to provide users with information services to meet the needs of cloud library platform to improve the operational efficiency and service capabilities.

\section{Cloud Library Management Platform}

Cloud library management platform layer is responsible for the management of service quality management, security performance management, authentication and login, storage access, resource allocation and deployment, remote process management. The key of library service platform is a cloud management platform management layer, platform layer service scope is the data file system, storage management system, development environment and parallel programming, distributed computing, cluster system management tools, application maintenance monitoring, management and operation of needed resources.

\section{Application Layer of Cloud Librar}

The highest level of cloud library service platform is the application layer. The application layer provides the user interface, the application layer to provide users with a unified mode of operation of a variety of business functions and interfaces, unified login / logout mode, according to the needs of different users, to provide the corresponding interface, verification and registration service management service in general. In the application layer, a cloud library service platform portal website, when user login portal home page, you can click on the operation, effective service.

Cloud library service platform in the process of building, can be established in the form of Web site, in the portal, the update service directory. The library cloud service platform, realize the connection of multiple services, to realize the 
optimization of cloud library service platform, cloud computing services to build cloud library service platform for each layer, each layer's function, can rely on cloud computing providers for the service platform, the number can dynamically increase or decrease hardware and software infrastructure.

\section{CHARACTERISTICS OF CLOUD LIBRARY}

\section{A. Large Data Storage in the Cloud Library}

In the cloud computing and big data environment, data source library, more than the total, the complex types of cloud storage has safe and reliable, low cost, easy expansion, resource control and other advantages, is the ideal choice for large data cloud library distributed cloud storage. Including private cloud and public cloud two kinds, public cloud flexibility and good scalability, private cloud to facilitate the control of better security. Cloud library data points to the internal security data and shared data, select the hybrid cloud storage model is more convenient and existing infrastructure combined to improve the efficiency of library storage of large data. The secret data of cloud library internal private cloud storage, the establishment of private cloud library within the data center, providing LAN data access, upload and download; sharing of data stored in the public cloud server, through the network access, Authors and Affiliations. Bibliographic data, cloud library free open access resources, data backup, data distributed access for public cloud storage, business data, user data, database and database resources for the purchase of private cloud storage. Cloud Library in the use of public cloud to retain the form of private cloud hybrid cloud information resources construction, the security risk can be reduced to a minimum degree.

Bibliographic data, cloud library free open access resources, data backup, data distributed access for public cloud storage, business data, user data, database and database resources for the purchase of private cloud storage. Cloud Library in the use of public cloud to retain the form of private cloud hybrid cloud information resources construction, the security risk can be reduced to a minimum degree.

\section{B. Large Data Processing of Cloud Library}

Cloud library big data distributed storage, data processing complex, massive storage in the data processing has timeliness requirements, is the big data processing layer of cloud library needs to solve the problem. Selection of cloud computing technology to handle large data, such as Map Reduce, query decomposition and traditional data analysis for distributed processing, the processing tasks assigned to different processing nodes, scheduling and processing tasks involved in the handling of data between a plurality of processing nodes, to realize the optimization of processing and storage resources of global computing. The working principle is to make the way of data processing, namely "Map decomposition", the data is divided into several parts, the distribution of multiple processor parallel processing, Reduce is "combined", the processor after the summary of the results.
Cloud Library of many problems can be resolved through the Map Reduce, such as the analysis of resource usage, user logs, user behavior data and large-scale index.

\section{Large Data Analysis of Cloud Library}

Data environment is an important asset, processing, analysis of massive data in the value of large data is the ultimate goal. To solve the problem of large data storage and processing of cloud library, through large data analysis to obtain valuable information knowledge. Cloud Library of realtime data, large-scale data association, dynamic data and unstructured data, association analysis, clustering analysis, data mining, semantic analysis, prediction engine visual analysis method of large data modeling, emotion analysis, cloud computing is an ideal choice for large data analysis. Analysis of large data order hybrid cloud technology, the original stage analysis can be performed on the public cloud infrastructure, and then use the private cloud component with the preservation of the available data information to the library, to ensure data security.

Hadoop is currently the most popular platform for analysis of large data processing, mainly for the analysis of Web, IT view log system log semi structured data and social networks, images, audio files and other unstructured data, using the Hadoop cloud library platform can realize large-scale and non structural analysis of data.

\section{Cloud Library Management OBJect}

Cloud library provides intelligent digital and network information services for users, including regional library alliance knowledge sharing service, automatic answering service, personalized service, city public services, knowledge retrieval and discovery service, reference service, Virtual Learning Center, digital literature resources platform.

\section{A. Information Flow of Cloud Library Based on Internet of Things}

On the one hand refers to the flow of information in the process of book circulation and management in the process of data transfer, on the other hand refers to the book in circulation through the intelligent sensor terminal, monitoring and use of books for data transfer. Smart things cloud library "based on the information flow, rely on cloud library link network, so that many library information management as a whole, books to borrow, borrow to generate data in intelligent sensor terminal, physical space location information to determine the book library through the positioning system set, and the books return the information to the owners of books through the Internet, timely remind readers to return on time, readers can choose the nearest library to return the books, then lending library in the management information on the book has been marked in return, return on the way yet storage. And then through the third party logistics system, the book back to the original library, this process can be used to track the positioning system to track. 
B. Analysis on the Characteristics of the Information Flow of the Intelligent Cloud Library Based on Internet of Things

The sensing device for future networking operating platform need to store hundreds of millions in the mass of information collected at different time, and summary, resolution, statistics, backup of the information, which requires the growth elasticity of storage resources, network resources and large-scale parallel computing ability. The information flow characteristics of intelligent "cloud library" based on Internet of things presents two features: the personalized end sign and the mass of data traffic. On the one hand, each library carries the one and only signs, can be used as a sensing terminal independent, the smart chip in addition to carrying can not change the basic attribute information, also includes books used to identify the owner, book number, book storage location, library management and other information are relatively stable, in addition to borrow books and borrowing time, return time dynamic information; on the other hand, in the cloud Library Based on the Internet of things, every book is a perception unit, facing the massive library collection, the perception of each chip on the literature and carry many constant data and variable data, intelligent sensing equipment manage the massive books, showing a state of massive information management. The number of books, liquidity and book management, intelligent networking in the decision for the management of books, information perception must pass through the network transmission timely, large flow of information put forward higher requirements for the construction of cloud computing and library network transmission.

\section{DEVELOPMENT TREND OF ClOUd LiBRARY}

\section{A. To Carry Out the Personalized Information Service of Users}

Cloud library to provide users with exclusive personalized information services. Collect user information, subject direction and hobbies, establish personal files, to provide users with personalized service, strengthen the service function of cloud library. But also for information integration, the user's data analysis, processing and forecasting, for the user to push customized information. To provide users with quality services, improve the timeliness of information, improve the user's usage. To integrate and analyze user behavior data, to understand the needs of user groups, to predict the information behavior of different users, to provide personalized service with targeted and distinctive.

\section{B. Using Large Data to Analyze the Cloud Library Services}

The use of Internet technology, two-dimensional code technology, positioning labeling technology for cloud cloud library, library service analysis provides conditions for user generated in the library in the data analysis, further to provide quality services for users. Cloud library uses a new technology, which is conducive to the use of information and mining, in favor of the one-way communication of information to the direction of the dissemination of information, to the implicit information to the dominant information.
The use of big data, open cloud library data, data mining, data analysis, data visualization technology for library service virtualization, personalized, deep and intelligent to provide technical support. Cloud library in-depth analysis of large data collection, user data, business data and external data resources, user needs and business trend of the library, improve the quality of library services, improve the user experience.

\section{REFERENCES}

[1] Chang Yahong. Research on the privacy protection of library users in the network information monitoring environment. Library Journal.2015.11

[2] Jin Zhimin, Liu Xiangzhi, Cui Hongbin. Library information resources sharing model reconstruction and innovation services based on Cloud Computing: office automation. 2015.2

[3] Shen Ling, Tang Jiayu. The age of the cloud - cloud reading service of the library. The library community. 2015.06

[4] Yang Sanfang. "To explore the reform and innovation of Library Service under the Internet plus" Shanxi Library Journal. 2016.01.

[5] Chen Jin,Yang Tingxiao. The research of the large data service of Library Based on cloud computing. The library. 2016.01

[6] Chong Yang. Cloud service model architecture of Digital Library Based on cloud computing technology. Computer knowledge and technology. 2015.12

[7] LiuYili.Research on the cloud service platform of digital library. Electronictest.2016.03 